\title{
Microbial Aortitis with Aneurysm Formation
}

\author{
Richard Raghoo', Marleen J. Noeverman², Ronald Hoekstra ${ }^{2}$, Rob Geelkerken ${ }^{3}$, \\ Goos D. Laverman ${ }^{2}$ \\ ${ }^{1}$ Leiden University Medical Center, Department of Nuclear Medicine, Leiden, Netherlands \\ ${ }^{2}$ ZGT Hospital, Department of Internal Medicine, Almelo, Netherlands \\ ${ }^{3}$ MST Hospital, Department of Vascular Surgery, Enschede, Netherlands \\ Email: r.raghoo@lumc.nl
}

Received 26 August 2014; revised 2 September 2014; accepted 26 October 2014

Copyright @ 2014 by authors and Scientific Research Publishing Inc.

This work is licensed under the Creative Commons Attribution International License (CC BY).

http://creativecommons.org/licenses/by/4.0/

(c) () 0 pen Access

\begin{abstract}
A 68-year-old man who presented with periodic fever and generalized weakness was diagnosed with Enterococcus faecalis bacteraemia from an infected abdominal aneurysm. The aneurysm was new: aortic dilatation was absent a year before. In such patients the diagnosis "microbial aortitis" is more appropriate than "mycotic aneurysm". We discuss the pathophysiology, epidemiology, prognosis and treatment of this condition.
\end{abstract}

\section{Keywords}

Mycotic Aneurysm, Potential Diagnostic Clues, Enterococcus, Crawford-Procedure

\section{Introduction}

Infections of the bloodstream often lead to a straight-forward diagnosis, e.g. infective endocarditis, infection of endovascular prosthetic material or infection of a pre-existing aortic aneurysm.

Here, we want to draw attention to a case with an insidious presentation of a mycotic aneurysm with subfebrile temperature lasting for months, no potential diagnostic clues and an unexpected source of bloodstream infection in a previously healthy man.

\section{Report}

A 68-year-old man was admitted to the Internal Medicine ward with bacteraemia. One year ago he had been dependent on intermittent haemodialysis for two weeks (non-tunnelled central venous catheter, right internal jugular vein) because of prolonged post renal obstruction.

During that period he developed a bacteraemia with Enterococcus faecalis, which was treated as a catheter-related sepsis by removing the central venous line and subsequent treatment with 2 grams amoxicillin 6

How to cite this paper: Raghoo, R., Noeverman, M.J., Hoekstra, R., Geelkerken, R. and Laverman, G.D. (2014) Microbial Aortitis with Aneurysm Formation. World Journal of Cardiovascular Surgery, 4, 181-185. 
times a day intravenously for two weeks according to national guidelines [1]. The post renal acute renal failure, recovered well and was found to be caused by a T3 prostate carcinoma, for which he had received hormonal therapy (bicalutamide and leuprorelin).

At the current presentation the patient has experienced episodes of fever, followed by symptom-free episodes and a series of blood cultures were again all positive for Enterococcus faecalis, with the same antibiotic resistance pattern as previously.

During this second admission, the patient was asymptomatic and a detailed patient history revealed no diagnostic clues; he did not suffer from weight loss, dyspnoea, back pain, complaints of the joints or complaints of increased miction. Physical examination revealed that our patient was not acutely ill, with a body temperature of $36.2^{\circ} \mathrm{C}$, a normal central venous pressure, blood pressure of $130 / 62 \mathrm{mmHg}$ and a regular heart rate of 62 beats per minute. His oxygen saturation, measured by pulse oximeter, was $100 \%$ and the respiratory rate was not increased. Furthermore, he did not show any signs of infective endocarditis, including heart murmurs. Examination of the abdomen was also unremarkable. No signs of peripheral oedema, skin defects, spinal column abnormalities, arthritis or prostatitis were present.

Laboratory evaluation (Table 1) showed mildly increased inflammatory parameters, normal liver enzymes and a slightly impaired kidney function. Urine dipstick analysis indicated a trace amount of erythrocytes and no sign of leukocytes. A chest X-ray was performed and showed no abnormalities.

The patient was admitted with an Enterococcus bacteraemia of unknown origin. Under clinical suspicion of infective endocarditis, or possibly blood stream infection from a source in the urinary or gastrointestinal tract, antibiotic treatment was started according to the national Dutch guidelines for endocarditis with this particular pathogen: amoxicillin 6 times a day 2 gr i.v. and gentamicin once a day $160 \mathrm{mg}$ [1].

Ultrasound examination of the heart, both transthoracic and transoesophageal, showed no heart valve disorders. The urinary culture showed no growth. During his admission our patient experienced no gastro-intestinal complaints, nor did he develop any fever.

Because an abdominal ultrasound examination a year earlier had showed a normal aorta (diameter of $1.7 \mathrm{~cm}$ ) and because of lack of any gastro-intestinal symptoms, further examination by colonoscopy or abdominal ultrasound examination were considered unlikely to demonstrate the source of infection. Therefore, a PET-CT scan was performed on the 12th day of admittance. The scan revealed an aneurysm of the abdominal aorta (AAA) with a diameter of $6 \mathrm{~cm}$ and an intense uptake of fluorodeoxyglucose at the same site (Figure 1). Thus the patient suffered from a "mycotic aneurysm" that must have developed within the past year.

The patient was transferred to the regional centre with thorax surgery facility.

During a 7-hour operation, with 17 litres of blood loss, he underwent a partial aorta resection with placement of a Dacron aortic graft via a transperitoneal "Crawford procedure”. Postoperatively he developed a Systemic Inflammatory Response Syndrome for which he received mechanical ventilation at the Intensive Care Unit for six days and he remained dependent on haemodialysis for 2 months. Before the operation the patient had been treated with amoxicillin for three weeks and post operatively this was continued for another 12 weeks (twice a day $2 \mathrm{~g}$ during the first 3 weeks, twice a day $1 \mathrm{~g}$ afterwards). Cultures obtained from the aneurysmatic thrombus showed no growth. Pathologic analysis revealed a severely arteriosclerotic vascular wall with a few gram posi-

Table 1. Blood analysis of patient A at admittance.

\begin{tabular}{|c|c|c|c|}
\hline Analysis & Patient A & Reference values & Unit \\
\hline $\mathrm{Hb}$ & 6.2 & $8.5-11$ & $\mathrm{mmol} / \mathrm{L}$ \\
\hline Erythrocyte sedimentation rate & 43 & $<20$ & $\mathrm{~mm} / \mathrm{h}$ \\
\hline CRP & 41 & $<10$ & $\mathrm{mg} / \mathrm{L}$ \\
\hline Leukocytes & 4.6 & $4.0-10$ & $10^{9} / \mathrm{L}$ \\
\hline Thrombocytes & 238 & $150-400$ & $10^{9} / \mathrm{L}$ \\
\hline Urea & 5.7 & $2.9-7.5$ & $\mathrm{mmol} / \mathrm{L}$ \\
\hline Creatinine & 109 & $65-105$ & $\mu \mathrm{mol} / \mathrm{L}$ \\
\hline ASAT & 24 & $<40$ & $\mathrm{U} / \mathrm{L}$ \\
\hline ALAT & 8 & $<45$ & $\mathrm{U} / \mathrm{L}$ \\
\hline $\mathrm{LDH}$ & 236 & $<250$ & $\mathrm{U} / \mathrm{L}$ \\
\hline
\end{tabular}




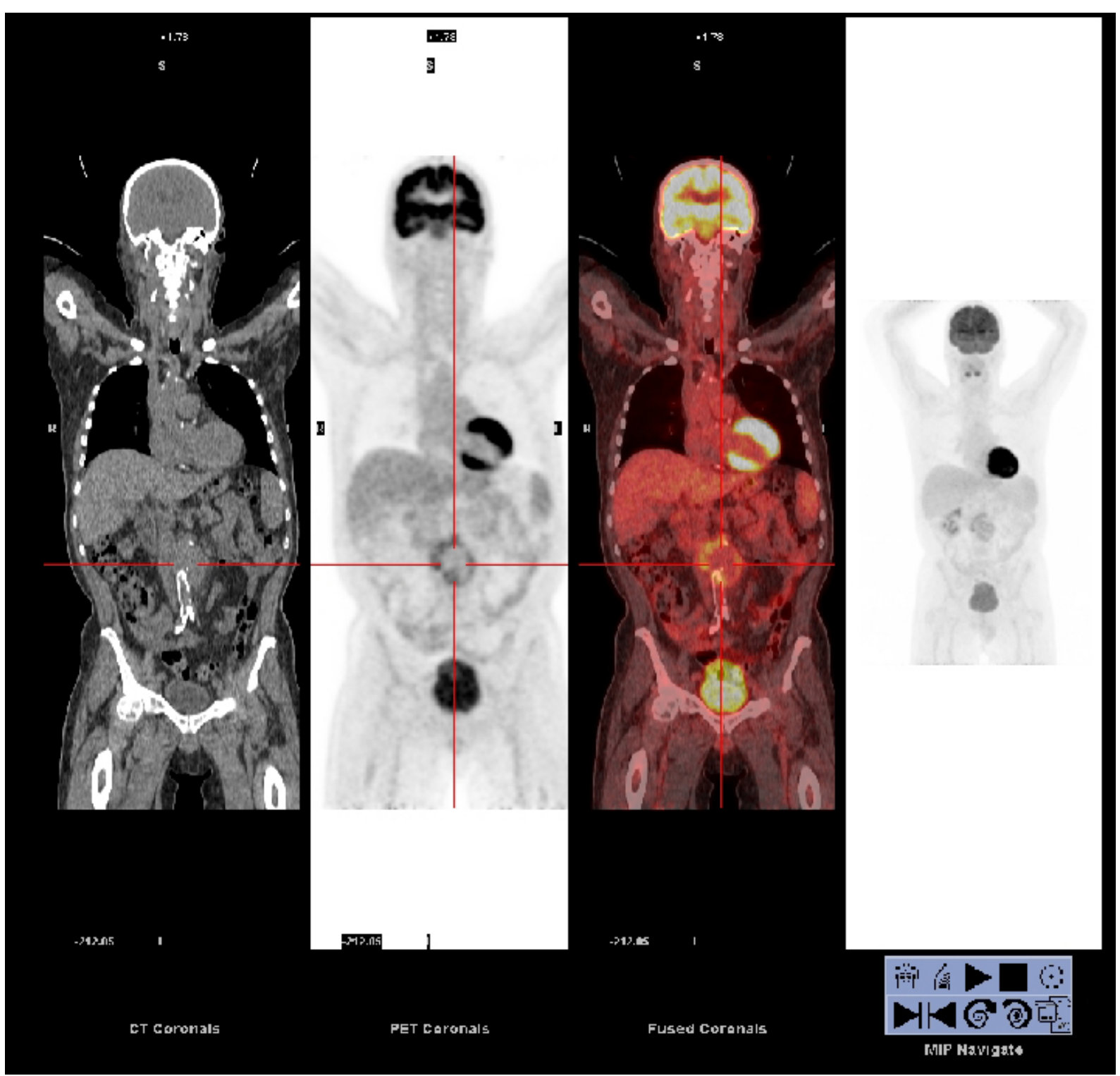

Figure 1. PET-CT scan of patient A. Coronal images displaying increased metabolic uptake at the site of the abdominal aortic aneurysm.

tive bacteria, though not convincingly located in the tissue itself. Two months after the surgical procedure he was discharged to his home in relative good health.

\section{Discussion}

We present a patient with an Enterococcus bacteraemia from an infected abdominal aneurysm.

In contrast with our initial hypothesis, there was no endocarditis. According to the Duke Criteria for Infectious Endocarditis [2] there was only 1 major criteria (two separate Blood Cultures positive for causative organisms) and possibly 1 minor criteria (fever $>38$ degrees Celsius, measured at home) at presentation.

A diagnostic dilemma arose since there were no "potential diagnostic clues" for any other common source of a bacteraemia. Though there is little evidence supporting the use of dual-modality positron emission tomography/computed tomography (PET-CT) in bloodstream infection from unknown source, this was chosen as the next diagnostic step, and lead to the eventual diagnosis of a mycotic aneurysm. In addition to visualisation of inflammatory or malignant processes, the detection of infectious foci is an important property of the PET-CT. 
F-18-fludeoxyglucose (FDG) accumulates in metabolic active cells, including activated leukocytes at the site of an infection, thus making the inflammatory process itself visible [3] [4]. It was because of these properties that we chose PET-CT in this patient case. It must be emphasized however that simple abdominal ultrasound examination would also have led to the diagnosis.

Mycotic aneurysms are a rare ( $0.9 \%$ of all aneurysms) [5], but severe condition with a reported mortality risk of up to $44 \%$ [6]. Symptoms vary and usually include fever (75\%), abdominal pain and back pain (33\%), abdominal pain and fever (20\%), and often a palpable aneurysm (53\%) [7] The presentation can be insidious with a subfebrile temperature lasting for months. The majority of cases of mycotic aneurysm reported in the literature are caused by Salmonella spp and Staphylococcus aureus [8] [9], and the former is associated with a higher risk of rupturation [10] [11]. Therefore, both the clinical presentation (virtually without potential diagnostic clues) and the causative micro-organism in our patient are very uncommon. The gold standard for the treatment of a mycotic aneurysm is prolonged antibiotic treatment and surgical resection with debridement of the infected aorta and surrounding tissue, covering up the infected area with muscles or the omentum, and placing a graft or extra-anatomical bypass [12] [13]. Even with adequate treatment mortality rates remain high with a postoperative mortality risk of $13.3 \%$ to $40 \%$ [12]-[15]. Our patient underwent a successful "Type IV thoracoabdominal aortic aneurysm repair" (Crawford-procedure).

The term "mycotic aneurysm" is somewhat confusing, and in our opinion not appropriate here. A mycotic aortic aneurysm develops when a pre-existing aneurysm becomes infected in case of a blood stream infection -irrespective of the source of initial infection. However, in our patient, abdominal ultrasonography performed a year before had shown that there was no aortic dilatation whatsoever, ruling out the possibility of secondary infection in an already existing arterial aneurysm. Most likely, he had developed a new aneurysm secondary to local destruction of the vascular wall caused by the bacterial infection per se. Therefore in cases like this, the term "microbial aortitis" (with the formation of an aneurysm) has been proposed as most appropriate [9].

Of note, there was a documented bacteraemia with the same micro-organism a year before, with exactly the same antibiotic resistance pattern. We propose that the bacteraemia from a year ago was insufficiently cleared from the endovascular compartment and that the microorganism somehow managed to stick to the aortic vascular wall, possibly facilitated by already existing abnormalities in the endothelium. As to the initial source of infection, considering the fact that the central venous catheter had been in situ for less than a week, a catheter-related sepsis seems unlikely. Considering the particular micro-organism, a urinary tract infection based on prostatitis could have been a possible focus, although urine cultures were negative at the time.

\section{Conclusion}

Looking for the source is important in the patient with endovascular infection since it may have major therapeutic implications. The previous absence of abdominal aneurysm does not exclude the possibility of a "mycotic aneurysm" since vascular wall infection per se may result in a new aneurysm. The term "microbial aortitis" is more appropriate then.

\section{Note}

The use of personal information in this case report was approved by the patient.

\section{References}

[1] Verhagen, D.W.M., Feltz, Mvd., Plokker, H.W.M., Buiting, A.G.M., Tjoeng, M.M. and Meer, J.T.Mvd. (2003) Optimisation of the Antibiotic Guidelines in the Netherlands VII. SWAB Guidelines for Antimicrobial Therapy in Adult Patients with Infectious Endocarditis. Netherlands Journal of Medicine, 61, 421-429.

[2] Durack, D., Lukes, A. and Bright, D. (1994) New Criteria for Diagnosis of Infective Endocarditis: Utilization of Specific Echocardiographic Findings. Duke Endocarditis Service. The American Journal of Medicine, 96, 200-209. http://dx.doi.org/10.1016/0002-9343(94)90143-0

[3] Bleeker-Rovers, C.P. and Meer, J.W.Mvd. (2008) Diagnostiek bij febris e causa ignota. Nederlands Tijdscrift Voor Geneeskunde, 152, 869-873.

[4] Vos, F., Bleeker-Rovers, C., Corstens, F., Kullberg, B. and Oyen, W. (2006) FDG-PET for Imaging of Non-Osseous Infection and Inflammation. Quarterly Journal of Nuclear Medicine and Molecular Imaging, 50, 121-130.

[5] Chan, F.Y., Crawford, E.S., Coseli, J.S., Safi, H.J. and Williams, T.W. (1989) In Situ Prosthetic Graft Replacement for 
Mycotic Aneurysm of the Aorta. Annals of Thoracic Surgery, 47, 193-203. http://dx.doi.org/10.1016/0003-4975(89)90268-3

[6] Fillmore, A.J. and Valentine, R.J. (2003) Surgical Mortality in Patients with Infected Aortic Aneurysms. Journal of the American College of Surgeons, 196, 435-441. http://dx.doi.org/10.1016/S1072-7515(02)01607-1

[7] Asthana, S., Walker, D., Iyengar, S., Shafran, S. and Conly, J. (1989) Mycotic Aneurysm Presenting as Fever of Unknown Origin. Western Journal of Medicine, 150, 694-696.

[8] Davies, O., Thorburn, J. and Powel, P. (1978) Cryptic Mycotic Abdominal Aortic Aneurysms: Diagnosis and Management. American Journal of Surgery, 136, 96-101. http://dx.doi.org/10.1016/0002-9610(78)90207-6

[9] Valentine, R. and Chung, J. (2012) Primary Vascular Infection. Current Problems in Surgery, 49, 128-182. http://dx.doi.org/10.1067/j.cpsurg.2011.11.004

[10] Lane, G., Cochrane, A. and Fone, D. (1988) Salmonellalmycotic Abdominal-Aortic Aneurysm. The Medical Journal of Australia, 149, 95-97.

[11] Jarrett, F., Darling, R., Mundth, E. and Austen, W. (1977) The Management of Infected Arterial Aneurysms. Journal of Cardiovascular Surgery, 18, 361-366.

[12] Muller, B., Wegener, O., Grabitz, K., Pillny, M., Thomas, L. and Sandmann, W. (2001) Mycotic Aneurysms of the Thoracic and Abdominal Aorta and Iliac Arteries: Experience with Anatomic and Extra Anatomic Repair in 33 Cases. Journal of Vascular Surgery, 33, 106-113. http://dx.doi.org/10.1067/mva.2001.110356

[13] Luo, C., Ko, W., Kan, C., Lin, P. and Yang, Y. (2003) In Situ Reconstruction of Septic Aortic Pseudoaneurysm Due to Salmonella or Streptococcus Microbial Aortitis: Long-Term Follow-Up. Journal of Vascular Surgery, 38, 975-982. http://dx.doi.org/10.1016/S0741-5214(03)00549-4

[14] Moneta, M., Taylor, L., Yeager, R., Edwards, J., Nicoloff, A., McConnell, D., et al. (1998) Surgical Treatment of Infected Aortic Aneurysm. American Journal of Surgery, 175, 396-399. http://dx.doi.org/10.1016/S0002-9610(98)00056-7

[15] Kyriakides, K., Kan, Y., Kerle, M., Cheshire, N., Mansfield, A. and Wolfe, J. (2004) 11-Year Experience with Anatomical and Extra-Anatomical Repair of Mycotic Aortic Aneurysms. European Journal of Vascular and Endovascular Surgery, 27, 585-589. http://dx.doi.org/10.1016/j.ejvs.2004.02.024. 
Scientific Research Publishing (SCIRP) is one of the largest Open Access journal publishers. It is currently publishing more than 200 open access, online, peer-reviewed journals covering a wide range of academic disciplines. SCIRP serves the worldwide academic communities and contributes to the progress and application of science with its publication.

Other selected journals from SCIRP are listed as below. Submit your manuscript to us via either submit@scirp.org or Online Submission Portal.
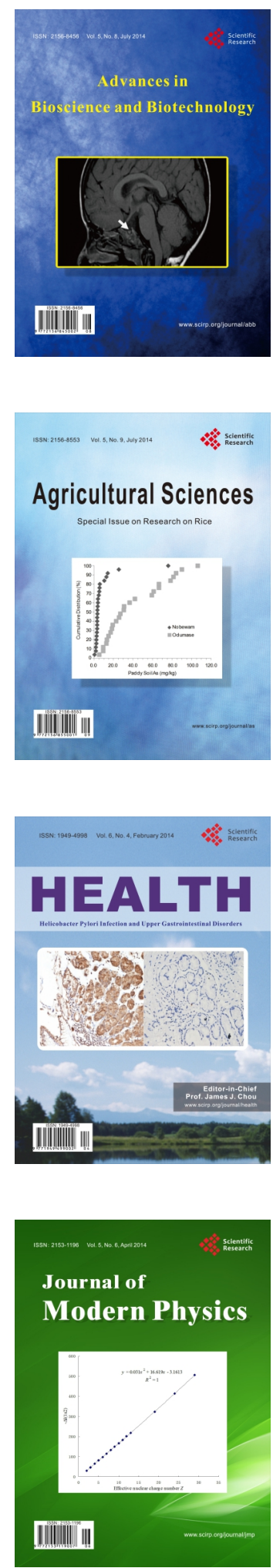
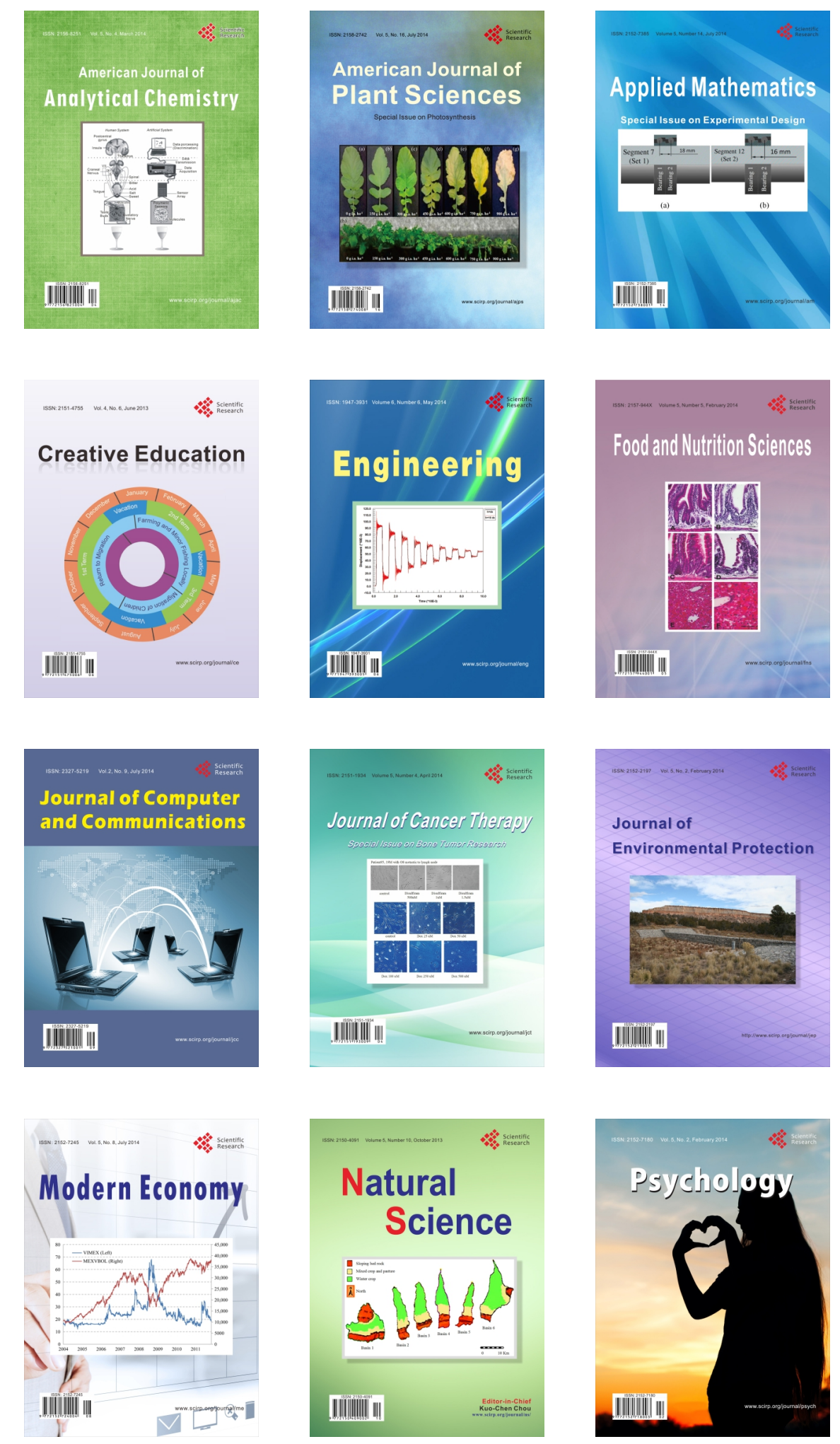